\title{
On Some Numbers Related to the Erdös-Szekeres Theorem
}

\author{
Mark J. Nielsen ${ }^{1}$, William Webb ${ }^{2}$ \\ ${ }^{1}$ Department of Mathematics, University of Idaho, Moscow City, Moscow \\ ${ }^{2}$ Department of Mathematics, Washington State University, Pullman, USA \\ Email: markn@uidaho.edu
}

Received January 15, 2013; revised February 16, 2013; accepted April 15, 2013

Copyright (C) 2013 Mark J. Nielsen, William Webb. This is an open access article distributed under the Creative Commons Attribution License, which permits unrestricted use, distribution, and reproduction in any medium, provided the original work is properly cited.

\begin{abstract}
A crossing family of segments is a collection of segments each pair of which crosses. Given positive integers $j$ and $k, \mathrm{a}(j, k)$ grid is the union of two pairwise-disjoint collections of segments (with $j$ and $k$ members, respectively) such that each segment in the first collection crosses all members of the other. Let $c(k)$ be the least integer such that any planar set of $c(k)$ points in general position generates a crossing family of $k$ segments. Also let $\#(j, k)$ be the least integer such that any planar set of $\#(j, k)$ points in general position generates a $(j, k)$-grid. We establish here the facts $9 \leq c(3) \leq 16$ and $\#(1,2)=8$.
\end{abstract}

Keywords: Erdos-Szekeres Theorem; Combinatorial Geometry

\section{Introduction}

For each positive integer $n \geq 3$ let $g(n)$ be the least integer such that every planar set of $g(n)$ points in general position contains the vertices of some convex $n$-gon. This number was introduced by Erdös and Szekeres in 1935 (see [1] and [2]) who established the bounds $2^{n-2}+1 \leq g(n) \leq\left(\begin{array}{c}2 n-4 \\ n-2\end{array}\right)+1$ and conjectured that the lower bound is in fact an equality. The values $g(3)=3$ and $g(4)=5$ are easy, and several proofs of the fact $g(5)=9$ have been given. However, no other values have been computed exactly and the upper bound given by Erdös and Szekeres stood until recently as the best known. A seqence of 1998 papers by Chung and Graham [3], Kleitman and Pachter [4], and finally Tóth and Valtr [5] improved the above-mentioned bound to

$$
2^{n-2}+1 \leq g(n) \leq\left(\begin{array}{c}
2 n-5 \\
n-2
\end{array}\right)+2
$$

Morris and Soltan [6] provide an excellent survey of related results.

Given the apparent difficulty of determining values of $g(n)$ we might well seek weakened notions of these numbers. For example, let $\mathcal{P}(n)$ be a combinatorial property satisfied by the vertex set of a convex $n$-gon. It might be interesting to ask how large a set $X$ must be to guarantee the existence of a subset of $X$ having property $\mathcal{P}(n)$. We will consider such generalizations where the property $\mathcal{P}(n)$ is a specified intersection behavior of some subset of the diagonals to a convex $n$-gon.

We will say that a set $X \subset \mathbb{R}^{2}$ generates a collection $\mathcal{S}$ of segments if each segment in $\mathcal{S}$ has its endpoints in $X$. Also, we will say that a collection $\mathcal{S}$ of segments is a crossing family if each pair of segments in $\mathcal{S}$ crosses (intersects at a point that is not endpoint to either segment). Now if $n \geq 2 k$ then the vertex set of a convex $n$-gon clearly generates a crossing family of size $k$. Define $c(k)$ to be the least integer such that any planar set of $c(k)$ points in general position generates a crossing family of $k$ segments. Then as we have noted, $c(k) \leq g(2 k)$, but we might expect $c(k)$ to be much less. Indeed, the main result in the paper by Aronov et al. [7] implies the much stronger bound

$$
c(k) \leq 12 k^{2}
$$

for these numbers. The authors of that paper ask whether 
this bound might be improved to a linear bound - a question that remains open at present.

Now let $j$ and $k$ be positive integers. Define a $(j, k)$-grid to be a collection of segments

$\left\{s_{1}, s_{2}, \cdots, s_{j}, t_{1}, t_{2}, \cdots, t_{k}\right\}$ such that each segment $s_{i}$ crosses each segment $t_{j}$ but such that segments $s_{i}$ and $s_{j}$ are disjoint if $i \neq j$, as are $t_{i}$ and $t_{j}$. If $n \geq 2 j+2 k$ then the vertex set of a convex $n$-gon clearly generates a $(j, k)$-grid. Define $\#(j, k)$ be the least integer such that any planar set of $\#(j, k)$ points in general position generates a $(j, k)$-grid. We again have the easy inequality $\#(j, k) \leq g(2 j+2 k)$, but a result of Nielsen and Sabo [8] implies the linear upper bound

$$
\#(j, k)<20 k(\text { when } j \leq k)
$$

for these numbers.

It appears at least superficially, then, that grids are easier to find than are crossing families, and that both are easier to find than are convex polygons. But while the progression from $g(n)$ to $c(k)$ to $\#(j, k)$ represents a geometric and computational simplification, none of these can be said to be simple. A look at the six-point cases illustrates the situation well.

(A) The value of $g(6)$ is not known. It is conjectured that $g(6)=17$, but $(\mathbf{0 . 1})$ gives only $17 \leq g(6) \leq 37$.

(B) The value of $c(3)$ is not known, but we will prove in this paper that $9 \leq c(3) \leq 16$.

(C) We will show below that $\#(1,2)=8$. However, this is the largest case for which the exact value of $\#(j, k)$ is known.

The purpose of this paper is to establish the facts mentioned in items (B) (see Section 1) and (C) (see Section 2 ). Bounds for some of the larger cases of these numbers will be given in a subsequent paper. The methods we use are not complicated, but some imagination is required to find an approach that reduces the number of cases to a manageable level.

\section{An Improved Bound for $c(3)$}

The bound (0.2) gives only $c(3) \leq 108$ - of course $c(3) \leq g(6) \leq 37$ (from (0.1)) is better. We are able here to improve this substantially to $9 \leq c(3) \leq 16$. We would be quite surprised if the actual value of $c(3)$ is not closer to the lower bound, but reducing the upper bound appears to be very difficult.

We begin by developing some notation that will be useful in the main proof. Let $X$ be a finite planar set in general position. Now let $A$ and $B$ be vertices of the convex hull of $X$ admitting parallel supporting lines. We may assume these supporting lines touch the convex hull of $X$ only at points $A$ and $B$ so that the points of $X \backslash\{A, B\}$ lie in the strip between them. One of the half-strips bounded by these lines and the segment $A B$ contains at least half the points of $X \backslash\{A, B\}$. Let this half-strip be called $\Sigma$, and let $m$ be the number of points of $X \backslash\{A, B\}$ in $\Sigma$. We define sequences of sets $\left\{X_{i}\right\},\left\{F_{i}\right\},\left\{V_{i}\right\}$, and $\left\{W_{i}\right\}(0 \leq i \leq m)$ as follows (see Figure 1).

- Let $X_{0}=X$.

- For each $i$ such that $X_{i}$ is defined let $F_{i}$ be the set consisting of those points in $\Sigma$ lying on the boundary of the convex hull of $X_{i}$, together with the points $A$ and $B$. Furthermore, set $V_{i}=X_{i} \backslash F_{i}$ and $W_{i}=X \backslash X_{i}$.

- Finally, for $i<m$ let $C_{i}$ be a point of $F_{i} \backslash\{A, B\}$ and define $X_{i+1}$ to be $X_{i} \backslash\left\{C_{i}\right\}$.

Note then that $\left|V_{i}\right|+\left|F_{i}\right|+\left|W_{i}\right|=|X|$ and that

$W_{i}=\left\{C_{0}, C_{1}, C_{2}, \cdots, C_{i-1}\right\}$, so $\left|W_{i}\right|=i$. We think of $F_{i}$ as being a "convex fence" separating $V_{i}$ and $W_{i}$.

More generally, we will say that a sequence of points $C_{1}, C_{2}, \cdots, C_{f}$ from $X$ is a $(v, w)$-fence for $X$ if

- $\quad X \backslash\left\{C_{1}, C_{2}, \cdots, C_{f}\right\}=V \cup W \quad$ where $|V|=v \quad$ and $|W|=w$,

- $C_{1}, \cdots, C_{f}$ are consecutive vertices on the convex hull of $V \cup\left\{C_{1}, \cdots, C_{f}\right\}$, and

- every segment joining a point of $V$ to a point of $W$ crosses one of the $f-1$ segments $C_{1} C_{2}, C_{2} C_{3}, \cdots, C_{f-1} C_{f}$.

In this case we say that $V$ is the set of points of $X$ inside the fence $C_{1} C_{2} \cdots C_{f}$ and $W$ is the of points of $X$ outside that fence.

Given positive integers $v, f$, and $w$ we will say that $X$ has property $\langle v / f / w\rangle$ if $X$ has a $\left(v^{\prime}, w^{\prime}\right)$ fence consisting of $f$ points for some $v^{\prime} \geq v$ and $w^{\prime} \geq w$. The sets described above yield a sequence of properties

$\left\langle v_{0} / f_{0} / 0\right\rangle,\left\langle v_{1} / f_{1} / 1\right\rangle,\left\langle v_{2} / f_{2} / 2\right\rangle, \cdots,\left\langle v_{m} / f_{m} / m\right\rangle$, for $X$ (where, of course, $v_{0} \geq v_{1} \geq v_{2} \geq \cdots \geq v_{m}$ and $f_{m}=2$ ).

Lemma 1.1. Let $X=\left\{P_{1}, P_{2}, P_{3}, Q_{1}, Q_{2}, Q_{3}, A, B\right\}$ where

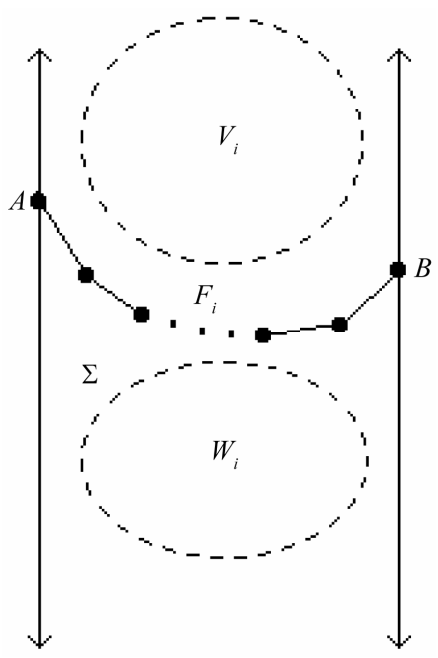

Figure 1. A convex fence. 
for each $i$ and $j$ the segment $P_{i} Q_{j}$ crosses $A B$. Then $X$ generates a crossing family of three segments including $A B$.

Clearly it is enough to show that $P_{i} P_{j} Q_{k} Q_{m}$ is a convex quadrilateral for some $i, j, k, m$, and to do this it is enough to show that line $P_{i} P_{j}$ misses segment $Q_{k} Q_{m}$ and line $\overline{Q_{k} Q_{m}}$ misses segment $P_{i} P_{j}$.

Note that the halfplane determined by $\overleftrightarrow{A B}$ and containing $\left\{Q_{1}, Q_{2}, Q_{3}\right\}$ is divided into four (or fewer) regions by the lines $\overleftrightarrow{P_{1} P_{2}}, \overrightarrow{P_{2} P_{3}}$, and $\overleftrightarrow{P_{1} P_{3}}$. Suppose first that one of these regions contains both $Q_{k}$ and $Q_{m}$. Now $\overrightarrow{Q_{k} Q_{m}}$ cannot intersect all three sides of the triangle $P_{1} P_{2} P_{3}$, so it misses some segment $P_{i} P_{j}$. From our observation above the points generate a crossing family of six segments.

If none of these regions contains two of the points of $\left\{Q_{1}, Q_{2}, Q_{3}\right\}$ then it must be the case that there is a segment $Q_{k} Q_{m}$ meeting exactly two of the lines $\overleftrightarrow{P_{1} P_{2}}$, $\overrightarrow{P_{2} P_{3}}, \overleftrightarrow{P_{1} P_{3}}$. But then $\overrightarrow{Q_{k} Q_{m}}$ cannot meet any of the sides of the triangle $P_{1} P_{2} P_{3}$ (since it cannot meet only one side of the triangle and there are two triangle sides that it clearly cannot meet, having crossed the lines determined by these sides at points outside of the triangle). But now $Q_{k} Q_{m}$ misses one of the lines $\overleftrightarrow{P_{i} P_{j}}$, so we once again have the situation described above.

Lemma 1.2. If a set $X$ has property $\langle v / f / w\rangle$ (with $v, w \geq 2$ and $f \geq 3$ ) and generates no crossing family of three segments then $X$ also has property $\langle v-2 / f-1 / w-2\rangle$.

Proof. Let $C_{1}, C_{2}, \cdots, C_{f}$ be a $(v, w)$-fence for $X$ with related components $V$ and $W$. Order the points of $V \bigcup\left\{C_{1}, \cdots, C_{f}\right\}$ radially from $C_{2}$ as

$\left\{P_{0}=C_{1}, P_{1}, P_{2}, \cdots, P_{v+f}=C_{3}\right\}$ (see Figure 2).

Now $X$ generates no crossing family of three segments by assumption, so by Lemma 1.1 there cannot be three points of $W$ on the same side $\Sigma$ of line $\overrightarrow{C_{2} P_{3}}$ as point $C_{1}$. (Again see Figure 2-the segment $C_{1} C_{2}$ is crossed by every segment joining such a point of $W$ to any one of $P_{1}, P_{2}$, or $P_{3}$.) But then $C_{2}, C_{3}, \cdots, C_{f}$ is a fence with related components $V \backslash\left\{P_{1}, P_{2}\right\}$ and $W \backslash \Sigma$.

Lemma 1.3. Any set having property

$\langle 2 f-1 / f / 2 f-1\rangle$ for some $f \geq 2$ generates a crossing family of three segments.

Proof. Assume to reach a contradiction that a set $X$

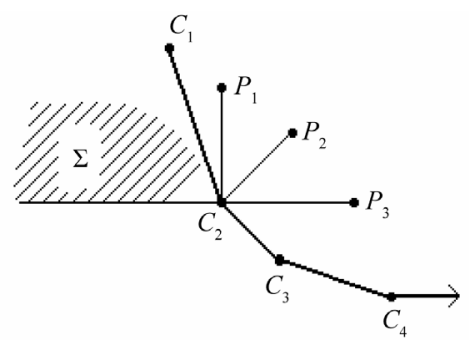

Figure 2. Illustrating the proof of Lemma 1.2. has property $\langle 2 f-1 / f / 2 f-1\rangle$ for some $f \geq 2$ but generates no crossing family of three segments. Then by Lemma $1.2 X$ also has property $\langle 3 / 2 / 3\rangle$. But by Lemma 1.1 any set with property $\langle 3 / 2 / 3\rangle$ will generate a crossing family of three segments - a contradiction.

These lemmas establish that a set generates a crossing family of three segments if it contains a subset with a $(3,3)$-fence of two points or a $(5,5)$-fence of three points. This fact will be used repeatedly in our next proof.

Theorem 1.4. $9 \leq c(3) \leq 16$.

Proof. The lower bound is established by considering the set of eight points depicted in Figure 3. (A few lines are shown to indicate relative positioning of the points.) We leave it to the reader to verify that this set fails to generate any crossing family of three segments.

Now let $X$ be any set of 16 points in general position. We will show that $X$ generates a crossing family of three segments. The construction given at the outset of this section established a sequence $\left\langle 16-f_{0} / f_{0} / 0\right\rangle$,

$\left\langle 15-f_{1} / f_{1} / 1\right\rangle,\left\langle 14-f_{2} / f_{2} / 2\right\rangle, \cdots$,

$\left\langle 16-m-f_{m} / f_{m} / m\right\rangle$ of properties for $X$. Consider the property $\left\langle 11-f_{5} / f_{5} / 5\right\rangle$.

We may clearly assume $f_{5} \leq 5$ else $X$ contains the vertices of a convex hexagon and thus clearly generates the desired crossing family. If $f_{5} \leq 3$ then we have either $\langle 8 / 2 / 5\rangle\langle 3 / 2 / 3\rangle$ or $\langle 7 / 3 / 5\rangle\langle 5 / 3 / 5\rangle$, either of which guarantees the crossing family by our preliminary lemmas. It remains, then, to examine the cases $f_{5}=4$ and $f_{5}=5$.

Case 1: $f_{5}=4$

In this case, $X$ has property $\langle 7 / 4 / 5\rangle$. As in Figure 4, let the fence be $A B C D$ and consider the four regions $R_{1}, R_{2}, R_{3}$, and $R_{4}$ as shown. Let $r_{i}$ denote the number of points of $X$ in region $R_{i}$, so that $r_{1}+r_{2}+r_{3}+r_{4}=7$.

If $r_{1}>0$ and $r_{3}>0$ (or similarly if $r_{2}>0$ and $r_{4}>0$ ) it is easy to see that $X$ then generates a crossing family of three segments (two of which are $A C$ and $B D$ ). So, if $r_{1}>$ 0 then we may assume either $r_{3}=r_{2}=0$ or $r_{3}=r_{4}=0$. This means either $A C D$ or $A B D$ is a $(7,5)$-fence, so our

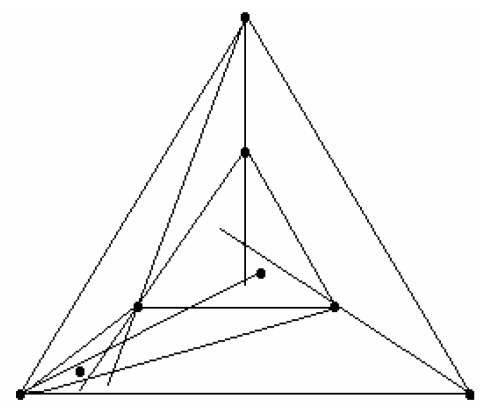

Figure 3. Example showing $c(3) \geq 9$. 


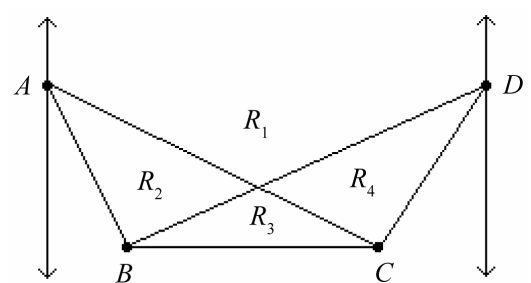

Figure 4. The general situation for Case 1.

preliminary lemmas would guarantee the desired crossing family for $X$. Thus, we may assume $r_{1}=r_{2}=0$ and $r_{3}+r_{4}=7$.

If $2 \leq r_{4} \leq 5$ then (using the points in $R_{3}$ and $R_{4}$ along with points $B$ and $D) A C$ is a $(3,3)$-fence. On the other hand, if $r_{4} \geq 6$ then $A C D$ is a $(5,5)$-fence. So (again using our lemmas) we may assume $r_{4} \leq 1$.

Subcase 1A: $r_{3}=6, r_{4}=1$

Order the points of $X$ in regions $R_{3}$ and $R_{4}$ as $P_{1}, P_{2}, \cdots, P_{7}$ radially from $B$ as in Figure 5. We may assume that the region $S_{1}$ in that figure (bounded by $\overrightarrow{A B}, \overleftrightarrow{P_{2} B}$, and the supporting line through $A$ to the convex hull of $X$ ) contains at most two points of $X$, else $A B$ is a $(3,3)$-fence (with the latter set of three points being $\left.\left\{P_{1}, P_{2}, D\right\}\right)$. But then $B C A$ is a $(5,5)$ fence where the points inside consist of the points in region $R_{3}$ less $P_{1}$ (if $P_{1} \in R_{3}$ ) and the points outside consist of the (at least three) points outside $A B C D$ not lying in $S_{1}$ together with $D$ and the point of $X$ in region $R_{4}$. By our preliminary lemmas, $X$ then generates a crossing family of three segments.

Subcase 1B: $r_{3}=7, r_{4}=0$

Consider now the region $S_{2}$ in Figure 6 (bounded by $\overleftrightarrow{A B}, \overleftrightarrow{P_{3} B}$, and the supporting line). If this region contains two points of $X$ then $B D$ is a $(3,3)$-fence where one set of three points is $\left\{P_{1}, P_{2}, P_{3}\right\}$ and the other consists of the two points in $S_{2}$ together with point $A$. Thus, we may assume $S_{2}$ contains no more than one point of $X$.

This shows that by discarding up to two points $\left(P_{1}\right.$ and $P_{2}$ ) inside and one point (in $S_{2}$ ) outside the fence $A B C D$ we can eliminate all segments (joining an inside point to an outside point) that cross $A B$. A mirror image of this same argument can be done for eliminating segments that cross $C D$. In this way we conclude that we can discard four points inside $A B C D$ and two points outside $A B C D$ and eliminate all segments except those that cross $B C$. So, $B C$ is a $(3,3)$-fence for the remaining subset of $X$. As before, this is sufficient to guarantee that $X$ generates the desired crossing family.

Case 2: $f_{5}=5$

In this case, $X$ has property $\langle 6 / 5 / 5\rangle$. Consider the convex hull of the five points making up the fence $A B C D E$. The diagonals of this pentagon determine eleven interior regions. If a point of $X$ lies in any of the

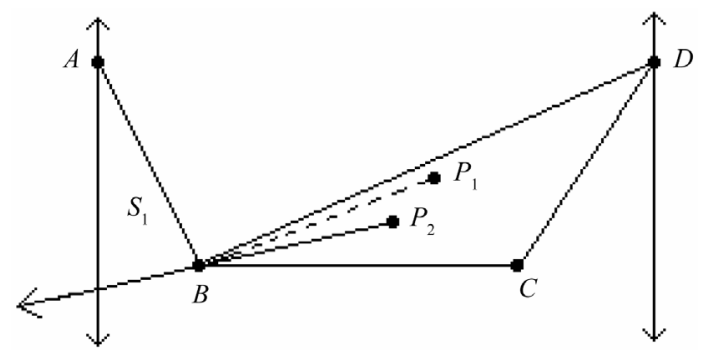

Figure 5. The arrangement for Subcase 1A.

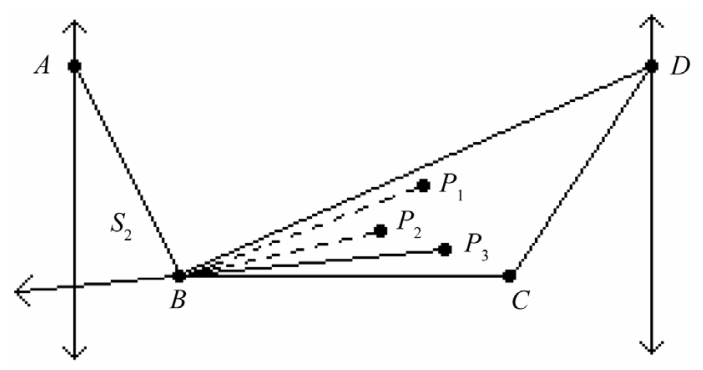

Figure 6. The arrangement for Subcase 1B.

five regions bounded by segments of the pentagon or in the central region bounded by all the diagonals, then $X$ generates a crossing family of three segments as shown in Figure 7.

Thus, we may assume that the six points of $X$ inside the fence $A B C D E$ lie in the five regions labeled $R_{1}$ through $R_{5}$ in Figure 8. As before, let $r_{i}$ denote the number of points of $X$ in region $R_{i}$.

- If points of $X$ lie in each of two adjacent regions from among $R_{1}$ through $R_{5}$ then it is easy to construct a crossing family of three segments (two are diagonals of $A B C D E$ and the other joins the points in question).

- If $r_{2}=r_{4}=0$ then $A C E$ is a $(6,5)$-fence-more than enough to guarantee the desired crossing family.

Putting together these two observations, we may now assume that $r_{2}>0, r_{1}=r_{3}=0$, either $r_{4}=0$ or $r_{5}=0$, and (of course) $r_{2}+r_{4}+r_{5}=6$.

- If $2 \leq r_{2} \leq 5$ then $A C$ is a $(3,3)$-fence where on one side we include two of the points from region $R_{2}$ along with $B$ and on the other side we include a point from $R_{4} \cup R_{5}$ along with $D$ and $E$.

- If $r_{2}=1$ then either $R_{4}$ or $R_{5}$ contains 5 points of $X$. If $R_{4}$ contains these points then $C E$ is a $(3,5)$-fence where the three points on one side consist of the point in $R_{2}$ together with $A$ and $B$. If the points lie in $R_{5}$ then similarly $A D$ is a $(3,5)$-fence.

Both of the above cases, then, lead to a crossing family of three segments generated by $X$. The only remaining case to consider is $r_{2}=6$ (and $r_{1}=r_{3}=r_{4}=r_{5}=0$ ). Here, $A B D E$ is a $(6,6)$-fence for $X$ (where we include $C$ with the points outside the fence). Note that Lemma 1.2 would allow us to conclude that either $X$ generates a crossing family of three segments or else its property 


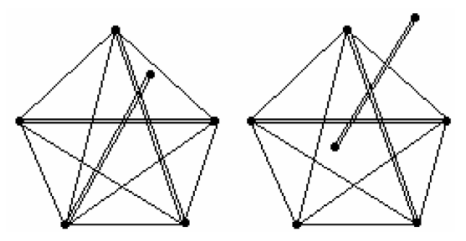

Figure 7. Crossing families in easy instances of Case 2.

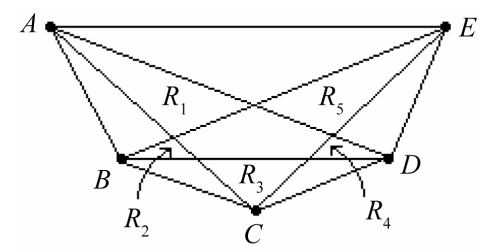

Figure 8. The arrangement for the remaining parts of Case 2.

$\langle 6 / 4 / 6\rangle$ must imply property $\langle 4 / 3 / 4\rangle$ (and

$\langle 2 / 2 / 2\rangle)$. Unfortunately, that is not sufficient under our lemmas to give the desired conclusion. Instead, we will need to be more careful in reducing the fence.

For our first step in the reduction, order the points of $X$ in region $R_{2}$ as $P_{1}$ through $P_{6}$ radially from $B$ as in Figure 9. We may assume the region $S_{1}$ in that figure contains no more than one point of $X$, since otherwise $B E$ is a $(3,3)$-fence (with two points from $S_{1}$ and $A$ on one side and $\left\{P_{1}, P_{2}, P_{3}\right\}$ on the other). Then discarding any point in $S_{1}$ along with $P_{1}$ and $P_{2}$, we may eliminate all segments (joining a point inside $A B D E$ to a point outside) that cross $A B$; all this while leaving at least four inside points and at least five outside points.

The second part of the reduction is accomplished by ordering the remaining points of $X$ in $R_{2}$ as $Q_{1}$ through $Q_{4}$ radially from $D$ as in Figure 10. We may assume the region labeled as $S_{2}$ in that figure contains no more than 2 points of $X$, else $D E$ is a $(3,3)$-fence (with three points from $S_{2}$ on one side and $\left\{A, Q_{1}, Q_{2}\right\}$ on the other). Thus, discarding points in $S_{2}$ along with $Q_{1}, B D$ is now a $(3,3)$-fence for the remaining set, and our reduction is complete.

We have demonstrated that in every possible case the set $X$ must generate a crossing family of three segments, so the theorem is proved.

\section{An Exact Value for \#(1,2)}

Here we prove the only exact value known for any of the six-point configuration numbers mentioned in the introduction. The following well-known fact will prove useful in the analysis.

Lemma 2.1: Suppose that $X=\left\{A, B, P_{1}, P_{2}, \cdots, P_{n}, Q_{1}, Q_{2}, \cdots, Q_{n}\right\}$ where for each $i$ and $j$ the segment $P_{i} Q_{j}$ crosses $A B$. Then $X$ generates a $(1, n)$-grid consisting of $n$ pairwise disjoint segments

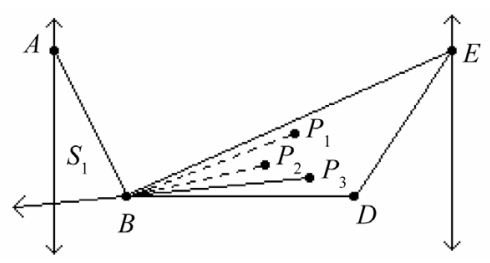

Figure 9. The first step in reducing the fence for Case 2.

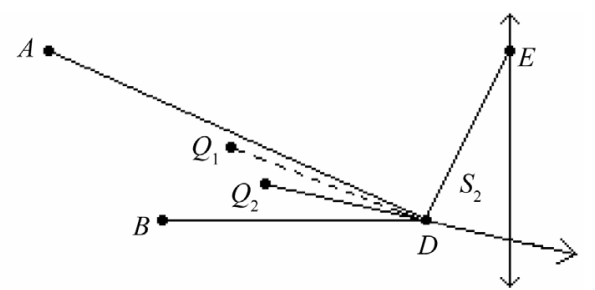

Figure 10. The second step in reducing the fence for Case 2.

each of which crosses $A B$.

Proof. Let $\pi$ be the permutation of $\{1,2, \cdots, n\}$ such that the sum of the lengths of the segments $P_{n} Q_{\pi(n)}$ is minimized. Then the segments

$\left\{A B, P_{1} Q_{\pi(1)}, P_{2} Q_{\pi(2)}, \cdots, P_{n} Q_{\pi(n)}\right\}$ form a $(1, n)$-grid.

Theorem 2.2: $\#(1,2)=8$.

Proof. Figure 11 shows a set of seven points that generates no $(1,2)$-grids. (This is easily checked by hand.)

It remains to prove that any set of eight points in general position will generate a $(1,2)$-grid. Let $X$ be any such set, let $Z$ be a vertex of the convex hull of $X$, and let $X^{\prime}=X \backslash\{Z\}$. We consider several cases depending on the shape of the convex hull of $X^{\prime}$.

Case 1. If the convex hull of $X^{\prime}$ is a 6-gon or 7-gon then $X^{\prime}$ clearly generates a $(1,2)$-grid.

Case 2. Suppose the convex hull of $X^{\prime}$ is a 5-gon $A B C D E$ with the remaining points of $X^{\prime}$ being $P$ and $Q$. If $P$ and $Q$ lie on opposite sides of any diagonal to $A B C D E$ then $X^{\prime}$ generates a $(1,2)$-grid by Lemma 2.1 . Consequently, we may assume that $P$ and $Q$ lie in the "inner pentagon" determined by the diagonals. We may also assume that $Q$ is interior to the triangle $P A B$. But then $Q D$ crosses both the diagonal $C E$ and either $P A$ or $P B$, giving us a (1,2)-grid (see Figure 12).

Case 3. If the convex hull of $X^{\prime}$ is a quadrilateral $A B C D$ then the three points

$\{P, Q, R\}=X^{\prime} \backslash\{A, B, C, D\}$ must be distributed between the four regions determined by the diagonals of $A B C D$. By Lemma 2.1 we may assume that these points are not separated by either diagonal - thus all lie in the same region.

Assume then that $P, Q$, and $R$ are each interior to both triangles $A B C$ and $B C D$. If points $\{A, P, Q, R\}$ form the vertices of a convex quadrilateral then its diagonals together with segment $B D$ form a $(1,2)$-grid (see the left 


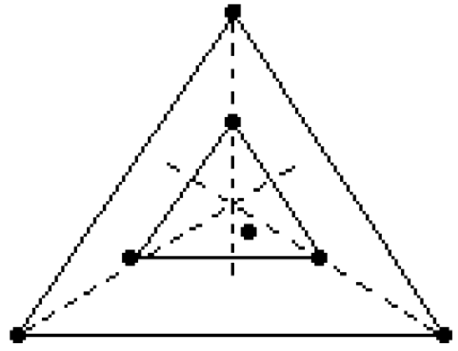

Figure 11. A set of seven points with no (1,2)-grid.

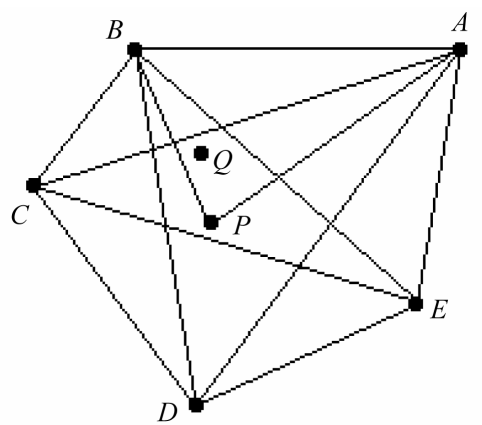

Figure 12. A (1,2)-grid for Case 2.

half of Figure 13). Thus, we may also assume that $R$ is interior to the triangle $A P Q$ as in the right half of Figure 13. We now consider some subcases. Recall that there is a point $Z$ of set $X$ lying outside of the convex hull of $X^{\prime}$. The segment $R Z$ must meet either $B C$ or one or both of the diagonals of $A B C D$.

Subcase 3A. Suppose first that $R Z$ meets $B C$. Note that it must also meet one of the sides of triangle $A P Q$, and that this side is disjoint from $B C$. Thus $X$ generates a $(1,2)$-grid in this case.

Subcase 3B. Next suppose that $R Z$ meets exactly one of the diagonals of $A B C D$. Then these diagonals together with $R Z$ form a $(1,2)$-grid.

Subcase 3C. Finally, suppose that $R Z$ meets both diagonals of $A B C D$. In particular, then, $R Z$ meets $B D$. But $B D$ meets both $A P$ and $A Q$, and $R Z$ must miss one of these. So, this case also yields a generated (1,2)-grid.

Case 4. The only remaining case is to assume that the convex hull of $X^{\prime}$ is a triangle, say $A B C$, with the four remaining points of $X^{\prime}$ interior to this triangle. If $Z A B C$ is a convex quadrilateral then by separating $B$ from the remaining seven points we reduce to one of the earlier cases (see Figure 14).

Thus we may assume that $C$ is interior to triangle $Z A B$ (so that the convex hull of $X$ is a triangle). We may clearly assume that a similar configuration results if any of the three vertices of this triangle are separated from the other seven points. In this case the set $X$ must be as pictured in Figure 15: the convex hull of $X$ is a triangle $Z_{1} Z_{2} Z_{3}$ and the convex hull of $X \backslash\left\{Z_{i}\right\}$ is a triangle with $C_{i}$ as the third vertex. Two points of $X$, say $P$ and
$Q$, must lie in the region common to the interiors of triangles $C_{1} Z_{2} Z_{3}, C_{2} Z_{1} Z_{3}$, and $C_{3} Z_{1} Z_{2}$. We consider two subcases for the placement of these points.

Subcase 4A. First it is possible that either $P$ or $Q$ is not interior to triangle $C_{1} C_{2} C_{3}$. Assume, for instance, that segment $C_{2} C_{3}$ separates $P$ from $Z_{1}$ as in Figure 16. In this case $P Z_{1}$ meets both $C_{2} C_{3}$ and one of $C_{1} Z_{2}$ or $C_{1} Z_{3}$, yielding a $(1,2)$-grid.

Subcase 4B. Finally, assume both $P$ and $Q$ lie interior to triangle $C_{1} C_{2} C_{3}$. The ray $\mathbf{P Q}$ must meet one of the sides of this triangle, say $C_{2} C_{3}$. Then $Q$ is interior to triangle $\mathrm{PC}_{2} \mathrm{C}_{3}$ so we have the configuration depicted in Figure 17. The segment $Q Z_{1}$ must now meet a side
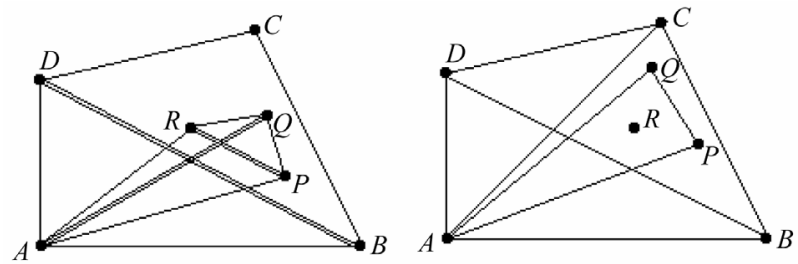

Figure 13. Possibilites for Case 3.

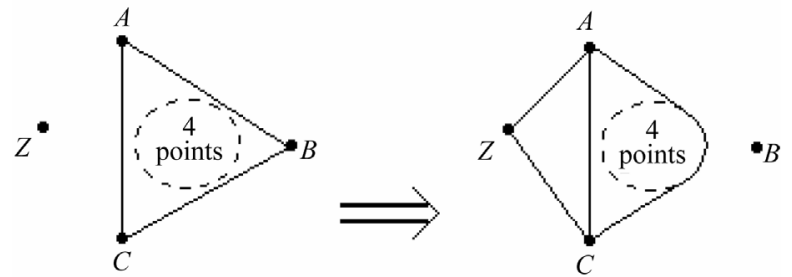

Figure 14. Reducing an instance of Case 4 to a previous case.

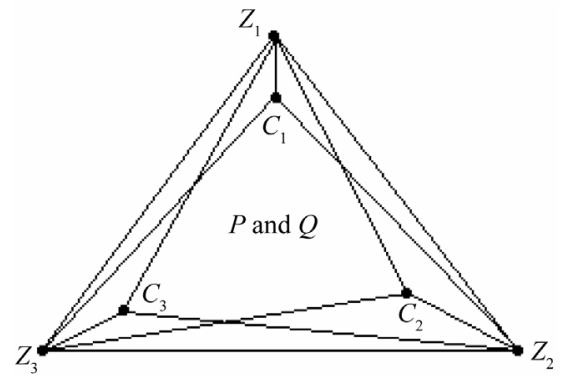

Figure 15. The difficult part of Case 4.

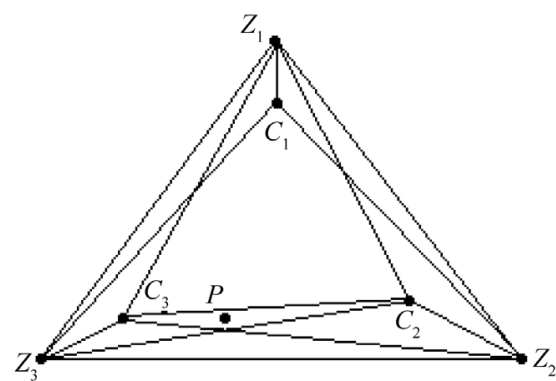

Figure 16. The arrangement for Subcase 4A. 


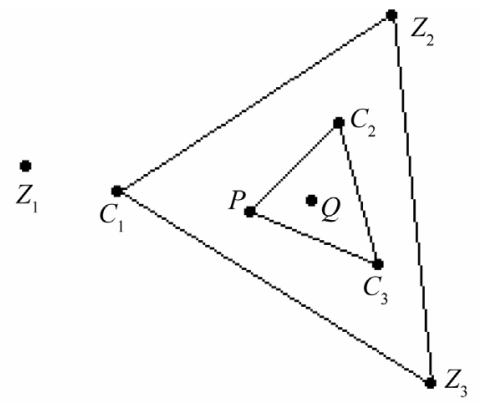

Figure 17. The arrangement for Subcase 4B.

of each of the triangles $C_{1} Z_{2} Z_{3}$ and $P C_{2} C_{3}$, again yielding a (1,2)-grid.

All cases have now been considered and the proof is complete.

\section{REFERENCES}

[1] P. Erdös and G. Szekeres, "A Combinatorial Problem in Geometry," Compositio Mathematica, Vol. 2, 1935, pp. 463-470. (Reprinted in: J. Spenceer, Ed., Paul Erdös: Selected Writings, MIT Press, Cambridge, 1973, pp. 3-12. Also Reprinted in: I. Gessel and G.-C. Rota, Eds., Classic Papers in Combinatorics, Birkhäuser, Basel, 1987, pp. 49-56.)

[2] P. Erdös and G. Szekeres, "On Some Extremum Problems in Elementary Geometry," Annales Universitatis Scientarium Budapestinensis de Rolando Eötvös Nominatae Sectio Mathematica, Vol. 3-4, No. 1, 1961, pp. 53-62. (Reprinted in: J. Spencer, Ed., Paul Erdös: The Art of Counting. Selected Writings, MIT Press, Cambridge, 1973, pp. 680-689.)

[3] F. R. L. Chung and R. L. Graham, "Forced Convex nGons in the Plane," Discrete \& Computational Geometry, Vol. 19, No. 3, 1998, pp. 367-371. doi:10.1007/PL00009353

[4] D. Kleitman and L. Pachter, "Finding Convex Sets among Points in the Plane," Discrete \& Computational Geometry, Vol. 19, No. 3, 1998, pp. 405-410. doi:10.1007/PL00009358

[5] G. Tóth and P. Valtr, "Note on the Erdös-Szekeres Theorem," Discrete \& Computational Geometry, Vol. 19, No. 3, 1998, pp. 457-459. doi:10.1007/PL00009363

[6] W. Morris and V. Soltan, "The Erdös-Szekeres Problem on Points in Convex Position-A Survey," Bulletin of the American Mathematical Society, Vol. 37, No. 4, 2000, pp. 437-458.

[7] B. Aronov, P. Erdös, W. Goddard, D. J. Kleitman, M. Klugerman, J. Pach and L. J. Schulman, "Crossing Families," Combinatorica, Vol. 14, No. 2, 1994, pp. 127-134. doi:10.1007/BF01215345

[8] M. J. Nielsen and D. E. Sabo, "Transverse Families of Matchings in the Plane," ARS Combinatoria, Vol. 55, No. 55, 2000, pp. 193-199. 